\title{
Mental Illness and Mass Shootings: A Quantitative Treatment of Risk Factors and Mitigation Strategies
}

\begin{abstract}
Jiahua $\mathrm{Wu}^{*}$
CUNY Kingsborough Community College, Brooklyn, NY 11235, United States

JIAHUA.WU04@students.kbcc.cuny.edu

ABSTRACT

This research paper aims at examining whether shooters with records of mental illnesses kill more people compared to mentally non-ill counterparts. The study also explores risk factors such as depression and schizophrenia, which might increase mass shootings' incidences. The research applies Python packages such as Seaborn, NumPy, and Matplotlib to visualize and categorize various mental illnesses that shooters experience. Independent samples' T-test was employed to observe whether shooters' prior signs of mental illness were related to the number of people they tried to kill. Results show that shooters have prior sign mental illness had higher values for the variable total victims $(\mathrm{M}=16.55, \mathrm{SD}=$ 14.34) than shooters without prior sign mental illness $(\mathrm{M}=11.82, \mathrm{SD}=8.91)$. The independent samples' $\mathrm{T}$-test indicated that the difference was statistically insignificant, $\mathrm{t}(41.32)=1.67, \mathrm{p}=.102,95 \%$ Confidence Interval $[-0.98,10.43]$. However, it is worth noting that the substantive significance (effect size) was calculated to be (0.46), which is a medium effect. Medium effect size indicates there might be a more significant relationship between the two variables.
\end{abstract}

Keywords: Mental Illness, Mass Shooting, Risk Factors for Gun Violence

\section{INTRODUCTION}

\subsection{Background}

In recent years, mass shooting has taken an epidemic proportion in the United States. Due to the convergence of factors such as a culture of gun ownership centered on the Second Amendment, the availability of guns, and the virtually absent regulation of gun ownership, the U.S. dominates the world in mass shootings. From 1966 to 2012, the US registered a record number of 270 Million guns and approximately 90 mass shooters. Americans own about 42 percent of firearms in the world [1]. According to Aizenman [2], the US has the $32^{\text {nd }}$ highest death rate from gun violence globally, with a record of 3.19 deaths in 100,000 people. The number of gun deaths per 100,000 in the United States is eight times higher than in Canada and 100 times higher than in the United Kingdom [2]. Moreover, a study conducted by mental health experts and police officers stated that about onethird of mass shootings in the 1990s were carried out by individuals with serious mental illnesses [3].

\subsection{Brief Summary}

The purpose of the current research is to examine whether shooters with prior signs of mental illness kill more people than non-mentally ill people. It evaluates whether differences in mental illness typologies, such as schizophrenia and depression, contribute to the severity of mass shootings. It explores the Mother Jones' Investigation dataset, which records mass shootings that happened in the U.S. from 1982 to May 2021. The methodology of this research is leveraging Python packages such as NumPy, seaborn, matpotlib to visualize and categorize various mental illnesses that shooters have. T-Test for independent samples was used to observe whether shooters' prior signs of mental illness were related to the number of people they tried to kill. Understanding that shooters' mental health history can be a risk factor to cause mass shootings can provide a better foundation for gun policy development. Meanwhile, psychiatrists assess whether mentally ill people are fit to carry guns may effectively reduce the level of casualties in mass shootings.

\section{LITERATURE REVIEW}

According to Lankford and Cowan's research, 25 percent of mass killers have a history of mental illness on their record [4]. Also, Lankford and Cowan [4] believe that further evidence suggests that almost all mass 
shooters have some kind of mental health problem, and the role of mental illness in a mass shooting is sometimes underestimated. According to FBI data, research that included 63 active shooters revealed that 25 percent of them had mental illnesses ranging from mild to severe mental health problems [5]. Buchanan et al. [5] found that schizophrenia patients had at least one harmful act throughout their 18-month follow-up, while patients with depression and psychosis had no injurious acts during their follow-up. Also, Beckett [5] finds that more than half of the mass shootings have been carried out by white people since the 1980s, but the proportion has declined in recent years, and the majority has tended to be black people.

According to Yelderman et al. [6], the severity of the person who has murdered people in a mass shooting, as well as his mental health history, is often linked to the scale of the mass shooting. Based on the research conducted by Yelderman et al. [6], a person's history of mental illness may affect his or her decision to become a shooter to a certain degree. Whenever this kind of person comes into touch with a gun or a weapon, their decisionmaking abilities are often impaired, and they may find themselves engaging in criminal activity without their awareness. At the same time, researchers also controlled different variables like age, race, location, the legality of weapons acquisition, and the use of assault rifles to investigate the correlation between the severity of the shooter's psychiatric history and the severity of the shooting [6].

Similarly, Hargarten advocates the classification of gun violence as a complex biopsychosocial disorder and understands and treats it with a multidisciplinary approach [7]. In addition, Vargas et al. [8] argue that in the aftermath of mass shootings, the proportion of people's search engines searching for mental illness and guns increases. Through analyzing the Mother Jones database, Philpott [9] demonstrates that since 1900, there have been nearly 200 mass shootings happened in the United States, and more than half of them have been committed by people who have been diagnosed with mental illness or who showed apparent prior signs of mental illness before the attacks. In contrast, Metzl and MacLeish [10] analyze the data from National Center for Health Statistics to derive the insights that there were 120,000 gun-related murders in the US between 2001 and 2010 , and less than $5 \%$ were committed by people with mental illness.

While insights such as Mother Jones's investigation, Mass Shooting Tracker, and National Center for Health Statistics can help in understanding the causal correlation between mental health history and mass shootings, such research like "Mass Shootings, Mental Illness, and Gun Control," "Using search engine data to gauge public interest in mental health, politics other and violence in the context of mass shootings," and "Mental illness, mass shootings, and the politics of American firearms" are ultimately constrained by their methodology. Indeed, on the one hand, researchers have used search engine data (Google Trends) to measure public concern about mental health and gun violence in the context of a mass shooting. Some of the limitations of this approach would be that the data in this study are based on 2004-2019, which is not real-time, and the analysis results may not be of value today. Moreover, current epidemic will affect people's search choices, that is, the public perception of gun violence alters the collected result. The researchers can expand the dataset they used, adding data of people who were not diagnosed with mental illness to increase data diversity and representativeness. Though with some limitations of the approach, Yelderman et al. [6] agree that shooters' mental health history severity is related to the severity of the mass shooting. As demonstrated by Yelderman et al. [6], some outcomes of this study can be potential for future research and help shape gun law legislation or restrict the use of guns to people with severe mental problems.

\section{METHODOLOGY}

\subsection{Data Source}

The current study analyzes data from Mother Jones's Investigation database to examine the relationship between mentally ill and non mentally ill shooters and the number of total victims. The Mother Jones's Investigation database developed the first-of-its-kind open-source database chronicling the United States mass shooting in July 2012, after the movie theater massacre in Aurora, Colorado. The Mother Jones's Investigation database record mass shootings that happened in the US from 1982 to 2021, and this database is still being updated [11]. The database records detailed information from the shooters' firearms use to their mental health condition, providing a unique insight into the phenomenon of gun violence. More importantly, Mother Jones's database is also available to the public.

\subsection{Method Adopting}

This research leveraging leverages Python packages such as NumPy, seaborn, matpotlib to visualize and categorize mental disorders that the shooters may have been suffering from. NumPy Python package helps perform mathematical operations in current research. Seaborn and Matpotlib are data visualization libraries in Python, which are used for making statistical graphics. In addition, the Datatab Statistics software was used to conduct the independent samples t-test and calculate the effect size. In the independent t-test, the total victim is a metric variable, and the prior sign mental health issue is a nominal variable. 


\section{RESULT}

The figures below visualize and categorize the various mental illnesses and help in the calculation of various factors that are involved. Figure 1 shows the factor that might affect the shooters are predominantly mental illness and unknown (89.9\%), followed by violent tendency $(6.4 \%)$ and environment stressor $(3.7 \%)$. We can estimate from the pie chart that shooters are predominantly mentally ill.

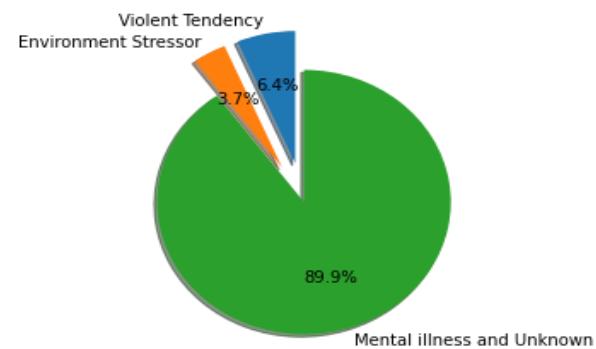

Figure 1 Factors that might affect shooters commit mass shootings
Breaking figure 1 down further, the highest percentage of factors that might affect the shooters to commit a mass shooting in figure 2 is unknown with roughly $45 \%$, followed by delusional disorder $(10.1 \%)$, uncategorized $(10.1 \%)$, depression $(10.1 \%)$, violent tendency (6.4\%), suicidal tendency (6.4\%), schizophrenia $(5.5 \%)$, environment stressors $(3.7 \%)$, PTSD (1.8\%), and Asperger syndrome (0.9\%). Based on these, the major mental illness factors of committing mass shootings are depression and delusional disorder. Skeem and Mulvey [12] point out that severe mental illnesses like schizophrenia, bipolar disorder, and major depression often impair the body's ability to function or perceive reality, and some mental conditions like delusions and hallucinations can directly provoke violent behavior. Moreover, based on the ECA study done by Swanson, the annual incidence of violence rate in people without mental illness or substance abuse was 2 percent, compared to 7 percent in people with mental illness [12]. Accordingly, we can assume that people with mental illness are more likely to be involved in violence than people without mental illness.

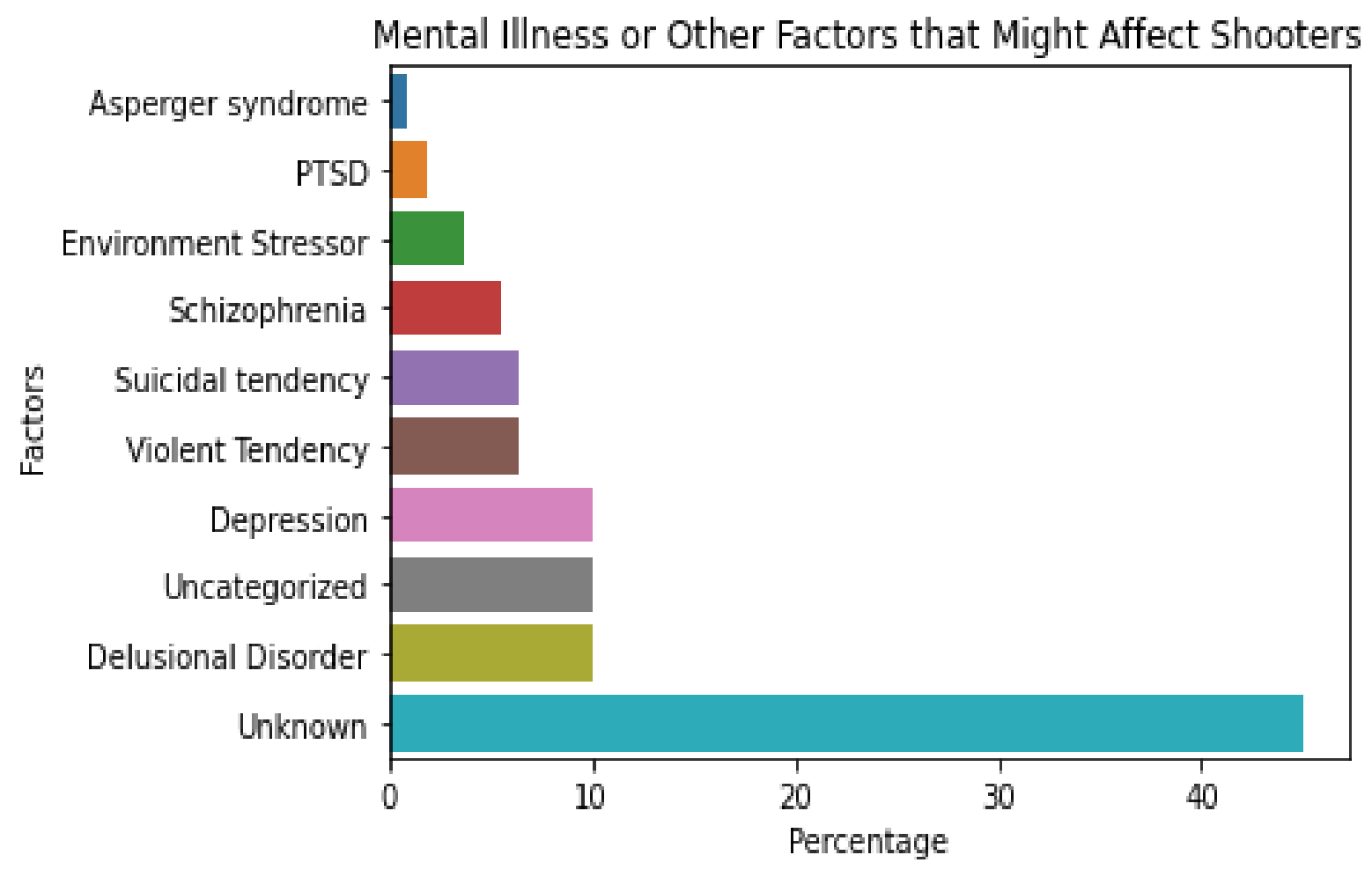

Figure 2 Types of mental illnesses and other risk factors that might affect shooters

Moreover, in addition to psychosis, which may trigger a shooter to commit a mass shooting, we should also look at the influence of non-psychotic factors such as violent tendencies and environmental stressors on the shooter. Demonstrated by Berkowitz and Alcantara [13] that some shooters in mass shootings have violent tendencies or criminal records, and more than half even kill themselves at the scene of the shooting. Significantly, as confirmed cases of COVID-19 surge again in the
United States, gun violence is also hitting the country. According to the FBI NICS firearm checks, the number of background checks for gun sales in 2020 increased 33\% in a single month from February to March, and the number of background checks in September 2020 already surpassed the total in 2019 [14]. Many people in the United States are currently under tremendous environmental and psychological pressure as a result of unemployment and the COVID-19 epidemic. The surge 
in guns has also increased the likelihood of mass shootings.

As shown in Figure 3 that shooters with prior sign mental illness had higher values for the variable total victims $(M=16.55, S D=14.34)$ than shooters without prior sign mental illness $(M=11.82, S D=8.91)$. A t-test for independent samples showed this difference was not statistically significant, $t(41.32)=1.67, p=.102$, with $95 \%$ Confidence Interval $[-0.98,10.43]$. However, it is worth noting that the effect size was calculated to be $d=.46$, which is a medium effect. There are 124 shooters cases in the Mother Jones database, which is a small database. Sullivan and Feinn [15] refer to effect size as the substantive significance and $\mathrm{p}$-value as statistical significance, and both the effect size and p-value are essential to help readers understand the magnitude of the differences and how they might be affected by the findings. Medium effect size indicates there might be a more significant relationship between the two variables. Meanwhile, a larger sample size is needed to estimate a more precise statistical value.

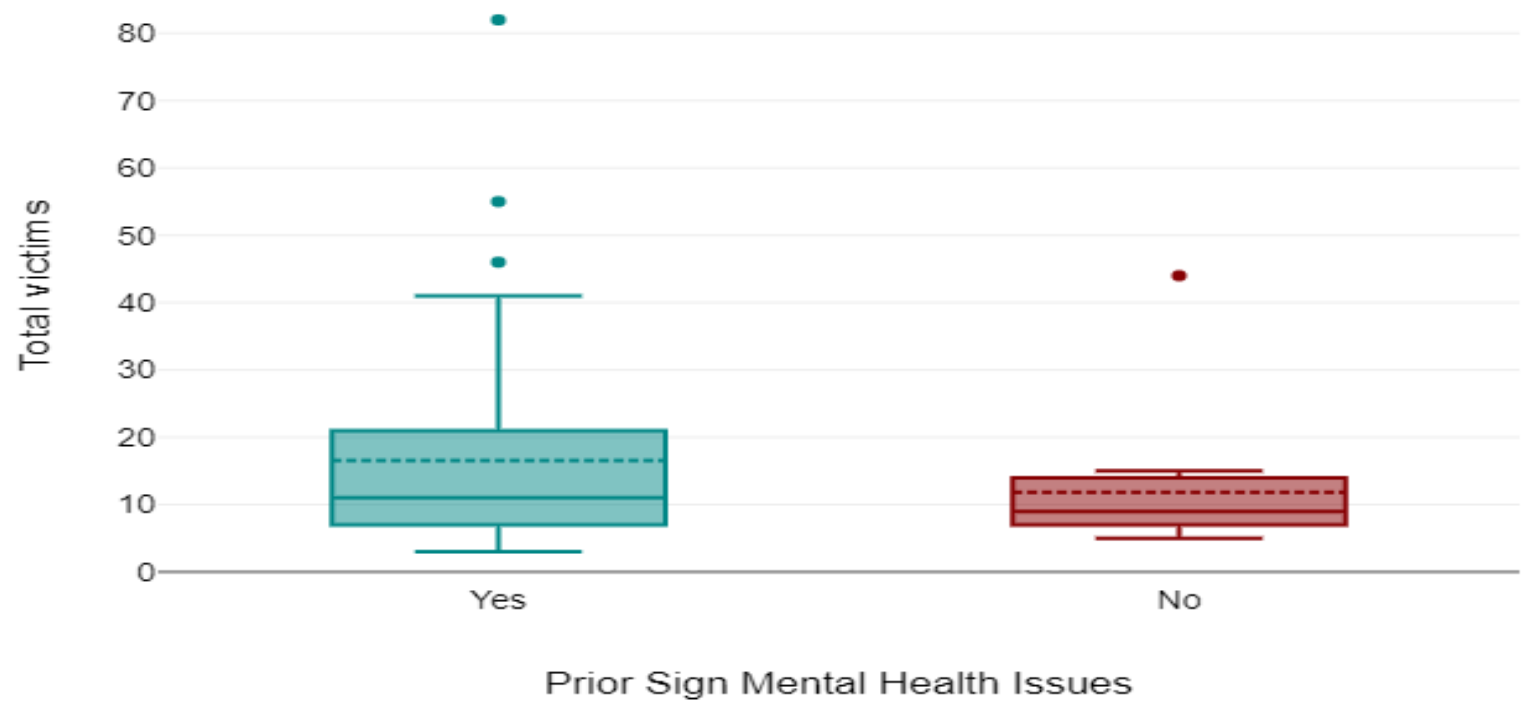

Figure 3 Results of independent samples t-test

\section{DISCUSSION}

There are some limitations of this research, which concerns the database, who reported the case, and the identification of the mental state. For the first limitation, the Mother Jones's Investigation database is still being updated, and adding more cases into it will create some uncertainty to the research and might impact and influence the hypothesis theory. For the second limitation, not all the mental illness cases in the database are reported by psychiatrists. Therefore, mental illnesses reported by professionals and families may yield different results. Moreover, there are a significant number of cases in Mother Jones's database where the shooters' mental states are unknown. Because of this, the existing data is not enough to indicate an accurate outcome of whether there is a relationship between the mental state and the number of victims.

Future research should include expanding the dataset, specifying each shooters' mental state, and adding another measure of severity of the mass shooting and cases from other countries. Expand the dataset by adding people who have not been diagnosed with mental illness to ensure that there is an increase in diversity and representation. Besides, it's also vital to ensure that every single shooter's mental state is identified. Moreover, adding another measure of severity of the mass shooting other than the number of victims makes it more compelling. More importantly, adding cases record from other countries would also increase the credibility of the upcoming research. Overall, these are some improvements that future research can corporate to enhance study result's accuracy.

\section{CONCLUSION}

The study aims to explore the factors that might drive shooters to commit mass shootings and propose mitigation strategies to ensure public safety. Greater knowledge of the connection that exists between shooters and mental disorders will also help to provide a stronger legal basis for the possession of firearms by the mentally ill.

With respect to public safety, it is important to understand whether and how different pathologies affect criminal intends and actions. Understanding the relationship between mental illness and shooters could result in better regulation of mentally ill people and gun 
possession. Being aware that a shooter's mental illness history is essential and can influence how they behave is the best way to ensure that guns are only issued to responsible individuals. It may also aid in developing gun policies that will benefit the public by reducing the risk factors. It is also necessary to involve psychiatrists to access mentally ill individuals to detect whether they can be trusted with firearms. Given the fact that these individuals have been shown to have a proclivity to act unintentionally, a comprehensive investigation should be conducted.

Politicians and news organizations should wait for the diagnosis of the mass shooter before making statements such as "a rational person could never commit such a thing." Psychological tests must be performed to evaluate a patient's psychiatric history to avoid perpetuating false preconceptions about the dangers of mental disorders [5]. Making such assumptions would exclude the possibility of determining alternative reasons for such acts, such as social disadvantages, exposure to domestic violence, anger and resentment directed towards specific people or groups, and a difficult economic situation, among others. In certain cases, the combination of such variables is more hazardous than the individual ones [5].

Additionally, it is necessary to raise public awareness of the political and socioeconomic factors that predict firearm violence. It is necessary to examine many aspects of weapon ownership in the United States to understand how the social, economic, cultural, and psychological factors may influence the gun violence trend [5]. This would also guarantee that guns are returned to individuals who are eligible while disqualifying those who seem to be a danger.

\section{REFERENCES}

[1] Fisher, M., \& Keller, J. (2017). Why Does the U.S. Have So Many Mass Shootings? Research Is Clear: Guns. - The New York Times. The New York Times - Breaking News, US News, World News and Videos.

https://www.nytimes.com/2017/11/07/world/ameri cas/mass-shootings-us-international.html

[2] Aizenman, N. (2021). Gun Violence Deaths: How The U.S. Compares With The Rest Of The World. NPR | WNYC.

https://www.npr.org/sections/goatsandsoda/2021/03 /24/980838151/gun-violence-deaths-how-the-u-scompares-to-the-rest-of-the-world

[3] Vestal, C. (2019). One-Third of Mass Shootings Committed by People With Mental Illness, Study Says | The Pew Charitable Trusts. The Pew Charitable Trusts | The Pew Charitable Trusts. https://www.pewtrusts.org/en/research-andanalysis/blogs/stateline/2019/08/08/one-third-of- mass-shootings-committed-by-people-with-mentalillness-study-says

[4] Lankford, A., \& Cowan, R. G. (2021). Has the role of mental health problems in mass shootings been significantly underestimated? Journal of Threat Assessment and Management, 7(3-4), 135-156. https://doi.org/10.1037/tam0000151

[5] Metzl, J. M., Piemonte, J., \& McKay, T. (2021). Mental illness, mass shootings, and the future of psychiatric research into American gun violence. Harvard review of psychiatry, 29(1), 81. Retrieved from https://www.ncbi.nlm.nih.gov/pmc/articles/PMC78 03479/

[6] Yelderman, L. A., Joseph, J. J., West, M. P., \& Butler, E. (2019). Mass Shootings in the United States: Understanding the Importance of Mental Health and Firearm Considerations. Psychology, Public Policy, and Law, 25(3), 212-223. https://doi.org/10.1037/law000020

[7] Hargarten, S. W., Lerner, E. B., Gorelick, M., Brasel, K., deRoon-Cassini, T., \& Kohlbeck, S. (2018). Gun Violence: A Biopsychosocial Disease. The Western Journal of Emergency Medicine, 19(6), 1024-1027. https://doi.org/10.5811/westjem.2018.7.38021

[8] Vargas, T., Schiffman, J., Lam, P. ., Kim, A., \& Mittal, V. . (2020). Using search engine data to gauge public interest in mental health, politics other and violence in the context of mass shootings. PloS One, $15(8)$, e0236157-e0236157. https://doi.org/10.1371/journal.pone.0236157

[9] Philpott-Jones, S. (2018). Mass Shootings, Mental Illness, and Gun Control. The Hastings Center Report, 48(2), 7-9. https://doi.org/10.1002/hast.832

[10] Metzl, J. M., \& MacLeish, K. T. (2015). Mental illness, mass shootings, and the politics of American firearms. American Journal of Public Health (1971), 105(2), 240-249. https://doi.org/10.2105/AJPH.2014.302242

[11] Follman, M., Aronsen, G., \& Pan, D. (2012, December 28). US mass shootings, 1982-2021: Data from Mother Jones' investigation. Mother Jones. https://www.motherjones.com/politics/2012/12/ma ss-shootings-mother-jones-full-data/

[12] Skeem, J., \& Mulvey, E. (2020). What role does serious mental illness play in mass shootings, and how should we address it? Criminology \& Public Policy, 19(1), 85-108. https://doi.org/10.1111/1745-9133.12473 
[13] Berkowitz, B., \& Alcantara, C. (2018). Mass shooting statistics in the United States - Washington Post. Washington Post; https://www.facebook.com/washingtonpost. https://www.washingtonpost.com/graphics/2018/na tional/mass-shootings-in-america/

[14] Kim, D.-Y., \& Phillips, S. W. (2021). When COVID-19 and guns meet: A rise in shootings. Journal of Criminal Justice, 73, 101783-101783. https://doi.org/10.1016/j.jcrimjus.2021.101783

[15] Sullivan, G. M., \& Feinn, R. (2012). Using Effect Size-or Why the P Value Is Not Enough. Journal of Graduate Medical Education, 4(3), 279-282. https://doi.org/10.4300/JGME-D-12-00156.1 